\title{
HYDRAULIQUE
}

\section{Recherche d'une méthode logique pour l'étude des aménagements hydrauliques pourvus de réservoirs saisonniers}

\author{
Par H. DE Folin, Ingénieur en chef des Ponts et Chaussées.
}

I. - On étudie souvent d'une façon qui nous semble trop simpliste les aménagements de force hydraulique pourvus de réservoirs saisonniers. On se donne les débits moyens mensuels d'une année dite "année moyennè ", d'une autre année dite "année sèche "; on recherche, par la méthode des débits cumulés, la façon dont on aurait manœuvré les réservoirs (ce qui est plutôt arbitraire, car cela suppose qu'au début de l'année on savait quel scrait le débit naturel de tous les mois suivants); l'année dite "sèche " étant isolée, on n'a d'ailleurs aucune donnée valable sur l'état où se trouveraient les réservoirs à son début. On arrive ainsi à quelques chiffres concrets de puissance, d'énergie, etc..., qui prétendent caractériser l'aménagement étudié. Mais c'est surtout quand il s'agit de comparer des variantes, que cette méthode apparaît rudimentaire, car les écarts des variantes peuvent être totalement inversés, croyons-nous, par l'application d'un procédé aussi fruste.

En cette matière, une seule méthode nous paraît donner une précision suffisante : supposer que l'aménagement a été construit quelque vingt ou trente ans en arrière (davantage si l'on veut et si l'on a des relevés assez anciens) et voir, au jour le jour, ce qu'il aurait donné (ce qu'auraient donné l'une et l'autre des variantes, si l'on fait une comparaison).

L'application de cette méthode-là suppose qu'à chaque moment de ce passé qu'on fait revivre, on sait comment on aurait manœuvré les réservoirs : par quels emprunts et avec quels apports. Cette idée, qu'il doit y avoir une "consigne " préalable de manœuvre des réservoirs saisonniers, a parfois surpris, nous la tenons cependant pour une évidence; dire le contraire, n'est-ce pas admettre que l'on manœuvrera les réservoirs " au petit bonheur "?... Dire que l'on ne peut avoir de consigne parce qu'on s'attachera au jour le jour à satisfaire les besoins d'énergie, n'est-ce pas méconnaître le fait que (sauf des accidents isolés et momentanés qui ne sont pas à prendre en compte en une étude générale) on peut - moyennant certaines hypothèses générales préalables - dresser à l'avance le "calendrier " des besoins d'énergie auxquels on devra satisfaire ?... Quelle sera la " consigne ", peu importe pour l'instant : l'essentiel est d'en constater l'existence et, dès lors, pour faire une étude vraiment "réaliste " de l'aménagement étudié, il suffit de supposer, non seulement que l'aménagement existe depuis quelque vingt ou trente ans, mais encore que l'on connaît sa " consigne " dès l'origine de cette période rétrospective.

Mais, dira-t-on, "vous allez faire de l'arbitraire : savez-vous si cette consigne sera la vraie ?... vos études, vos comparaisons seront ainsi viciées. "Oui, si on les présente comme une vérité absolue ; non, si on les présente en disant de quelle hypothèse et de quelle "consigne " elles sont fonction... Pour étendre la portée des études et des comparaisons, il suffira alors de multiplier les hypothèses sur la destination de la puissance et sur le "calendrier " des besoins en énergie ; sur les conditions de fonctionnement et de couplage de l'aménagement, etc..., de multiplier les consignes. Cela certes donnera du travail et un travail délicat, mais le propre d'un aménagement hydraulique est d'engloutir presque tout d'un coup un capital énorme qui, ensuite, produira sans efforts ni grands frais, pendant des années et peut-être des siècles, mais qui restera très lourdement chargé des intercalaires dus à une erreur initiale de conception. Si l'on pense davantage à cela, on ne reculera pas devant des études, si longues et complexes qu'elles soient, quand ces études peuvent permettre sérieusement d'approcher de la "bonne solution".

II. - L'emploi de cette méthode ne nous paraît d'ailleurs pas moins nécessaire, en dehors de l'étude générale des schémas, pour analyser de près tous les problèmes dédétail que pose l'aménagement et l'exploitation des forces hydrauliques : l'un des plus importants est celui des ententes et des plus-values, non encore sorti jusqu'à ce jour - nous semble-t-il — du domaine des dispositions législatives abstraites.

Supposons une concession A pourvue de réservoirs saisonniers et à l'aval de laquelle va être accordée une concession B, laquelle, par son cahier des charges, sera astreinte à une entente précise et concrète avec $\mathrm{A}$, c'est-à-dire en somme à des plus-values annuelles. Ajoutons, pour accroître l'intérêt du problème, que d'autres concessions antérieures C. D., etc..., existent déjà en aval de $A$ - d'ailleurs sans entente avec $A$ - et que les accords qui se concluront entre $A$ et $B$ serviront de base sérieuse, mutatis mutandis, pour les revendications que A pourra, en Conseil de Préfecture, exercer sur $C, D$, etc...

Va-t-on dire à $\mathrm{B}$ : "les réservoirs de $\mathrm{A}$ comportent une tranche utile $\mathrm{V}$, votre hauteur de chute est $\mathrm{H}$; la formule $\frac{2 \mathrm{VH}}{1.000}$ donne le nombre de KWH valorisés que vous procurera chaque année la concession $\mathrm{A}$, donc la mesure de l'annuité que vous devrez lui payer annuellement. " Ce serait supposer a priori que, chaque année, invariablement, A videra toute la tranche utile de ses réservoirs (et le fera d'ailleurs à un moment où cela intéressera B); la combinaison s'effondrerait en pratique dès la première année où les choses se passeraient autrement. Or, nous croyons que dire : " nous viderons chaque année - systématiquement et en totalité - la tranche utile des réservoirs de notre concession ", c'est manger son blé en herbe (à moins d'avoir un dispatcher doué du don de prophétie) et s'exposer aux pires déboires en fin d'année.

Va-t-on plus simplement faire payer à $B$, sur la base du mètre de chute à lui concédé, une part des dépenses dites " de régularisation "faites par A ?... On reconnaitra que c'est donner à $\mathrm{B}$ le droit de regard le plus large, et le plus mal défini d'ailleurs, sur ce que fera $\mathrm{A}$ : c'est "très élégamment. " remettre au lendemain les pires malentendus et les plus acharnées contestations, sans même laisser aux juges, en quelque chose d'aussi mal défini, le moindre fil conducteur leur permettant d'apercevoir la commune intention des parties !......

En désespoir de cause, se résoudra-t-on à ne fixer dans le Cahier des Charges de B que le " principe " de la plus-value - ce 

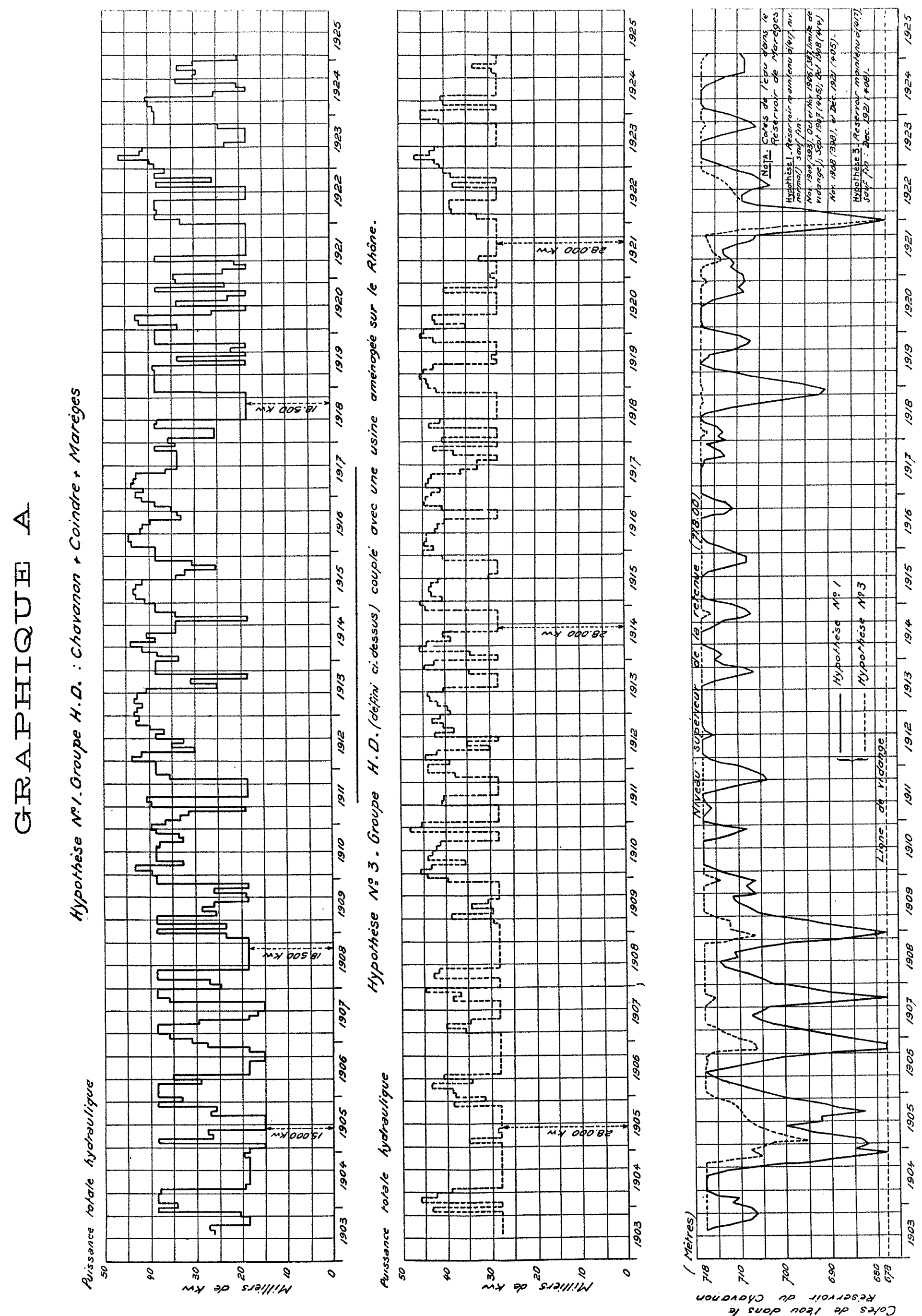

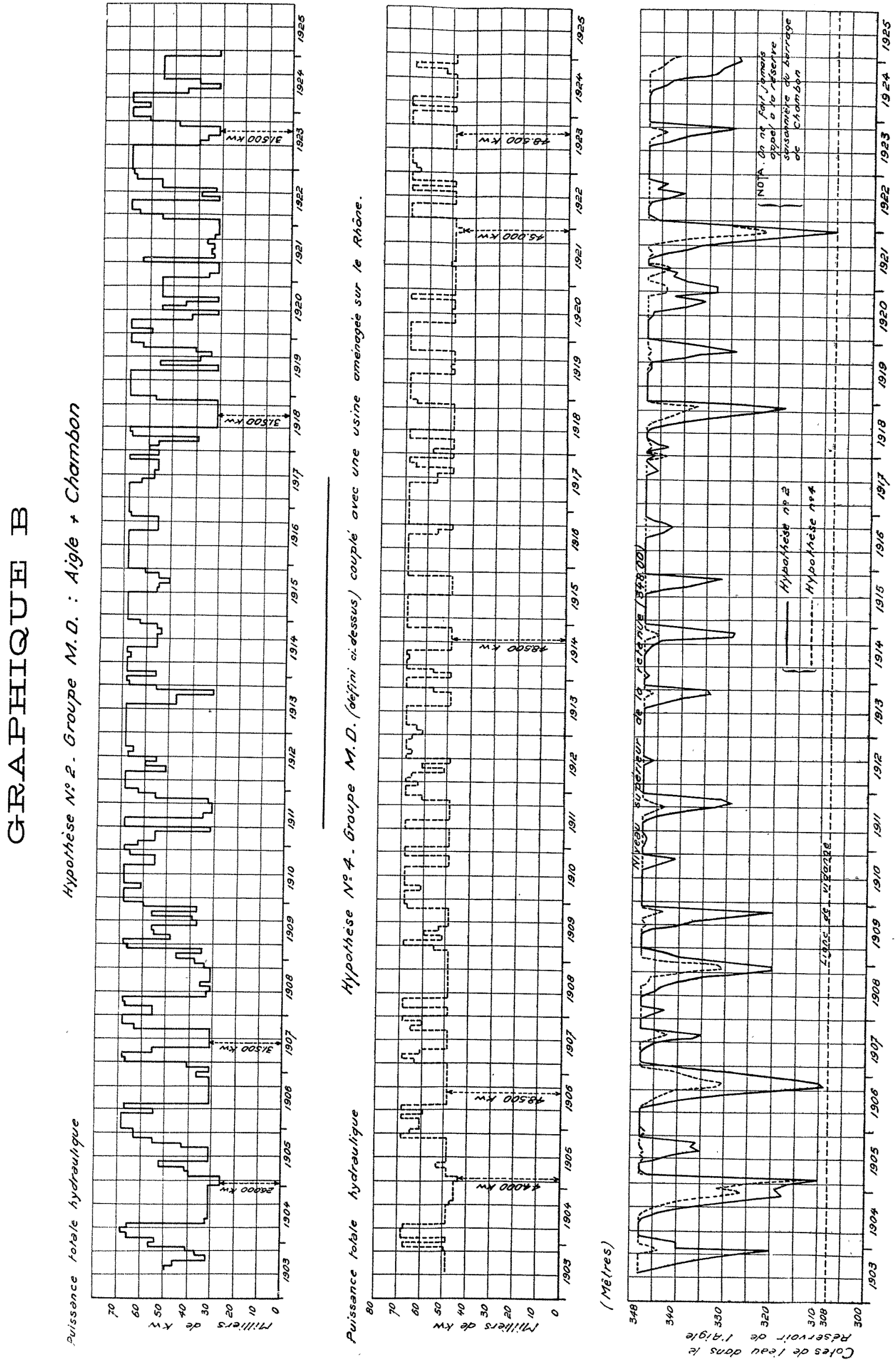
qui alors sera une simple redite de la loi - et à s'en remettre à des fixations ultérieures a posteriori ?... Nous croyons que ce sera fatalement une solution bien fâcheuse pour $\mathrm{A}$ et, du mème coup, pour les intérêts généraux du pays qui demandent la construction de réservoirs saisonniers, done qui veulent voir cette construction très sûrement encouragée et très sérieusement rémunérée. Car il nous paraît certain que l'on sera autrement armé pour faire consentir B à une entente précise et chiffréc a priori, au moment où l'on discute arec lui les textes de sa concession, que par la suite, quand il aura sa concession en poche, et qu'il s'arrangera - dans une fixation a posteriori - pour "démontrer" la soi-disant vanité des avantages à lui procurés par $\mathrm{A}$, sans que l'on puisse même en ce débat futur - après avoir remis au lendemain le soin de régler ce qu'on aura négligé de résoudre quand il le fallait et quand on le pouvait - prendre pour base de discussion d'autres " espèces" comparables : pour cela, en effet, il faudrait avoir réalisé au moins une fixation a priori.

Tout cela change de face si l'on a fait des études du genre de celles que nous préconisons et sil'on dit à $\mathrm{B}$ : «sur la base de telle " consigne " pour A, votre contribution annuelle sera de tant". L'amont, A, n'abdiquerait pas du tout la liberté de modifier sa consigne; le changement de consigne comporterait la révision de la plus-value, voilà tout. Sur cette révision, il n'est pas sûr qu'on se mette d'accord, mais on aura alors une chose essentielle à sa disposition en matière contentieuse, savoir une " espèce " comparable mutatis mutandis; une indication de la commune intention des parties; un fil conducteur permettant une solution précise et équitable, ce qui est en somme la seule chose indispensable et ce qui couvre l'amont aussi bien que l'aval.

En résumé, si l'aval s'engage à payer quelque chose de précis à l'amont, cela évidemment lui donne un droit de regard sur l'amont : on paie d'avance, ou l'on promet de payer, mais on veut en avoir pour son argent. Or nous croyons, comme nous l'avons dit au début de la présente étude, que l'amont marchera à l'aveuglette, " au petit bonheur ", s'il n'a pas de " consigne " et, si au contraire il a une consigne, l'aval est renseigné, couvert, garanti ; il peut payer sur le vu de cette consigne et des résultats qu'elle lui donne. La consigne, nécessaire au seul point de vue des intérêts de l'amont, est en même temps la sauvegarde de l'aval, donc la base des plus-values. Le changement de consigne comportera la revision de la plus-value; il y aura une tranche d'années pendant lesquelles la plus-value sera payée sur telle base, puis une autre tranche d'années correspondant à une autre base..., mais, dans chaque tranche, régime stable et très clair, bien défini, dans lequel chacun saura ce qu'il fait, ce à quoi il a droit, ce qui l'attend, "ce qu'il aura pour son argent ", sans passer le temps à se quereller au jour le jour sur la portée du droit de regard donné par la plus-value.

Les mêmes méthodes nous paraissent, pour les mêmes raisons, devoir être à la clef de toutes les autres ententes que pourra imposer l'Administration, au point de vue des échanges de courant et d'une façon générale de la meilleure utilisation de l'énergie d'ensemble, entre concessions d'un même bassin (loi du 16 octobre 1919) ; entre usines"d'une même région (y compris les thermiques existantes) réunies ou à réunir par un réseau de transport à haute tension (loi du 19 juillet 1922); à la clef de celles qu'elle pourra imposer entre usines thermiques projetées et usines hydrauliques, le jour impatiemment attendu par plus d'un où la loi soumettra à de telles ententes préalables la mise en service de toute nouvelle centrale thermique de quelque importance.

III. - Mais, quelle forme auront donc les "consignes " en question ?... Nous les voyons comme un système de tables à ntrées multiples, d'abaques, ou d'équations à multiples paramètres; les paramètres pourront être la date de l'année, l'état des réservoirs, l'état des glaciers, celui des neiges, le volume des pluies tombées pendant telle période, le débit naturel de tels cours d'eau, la puissance donnée par telles usines avec lesquelles on suppose se coupler dans telles ou telles conditions, le " calendrier des besoins d'énergie à satisfaire ", etc..., etc... Pratiquement, les variables à déterminer se ramènent aux niveaux à obtenir dans les réservoirs de l'aménagement étudié car ceux-ci déterminés, on connaît les débits artificiels des usines, donc la puissance fournie par elles.

Nous croyons que ces consignes seront extrêmement complexes et variées, qu'elles diffèreront profondément avec les régions, etc... Celles que nous avons imaginées dans les exemples simples ci-après n'ont donc aucunement pour but de figer les choses, mème dans les cas étudiés, mais au contraire de servir de point de départ pour des recherches bien plus fouillées, pour des discussions bien plus riches en idées et bien plus fécondes; c'est d'ailleurs là le seul objet de la présente note.

Exemples. - Ils sont pris sur la Dordogne supposẻe aménagée partiellement en deux groupes distincts et fonctionnant séparément : l'un dit Haute Dordogne (H. D.) et comprenant les trois chutes du Chavanon, de Coindre et de Marèges (usine-barrage de $69 \mathrm{~m}$. à $4 \mathrm{~km}$. environ en amont du pont de Vernéjoux) ;

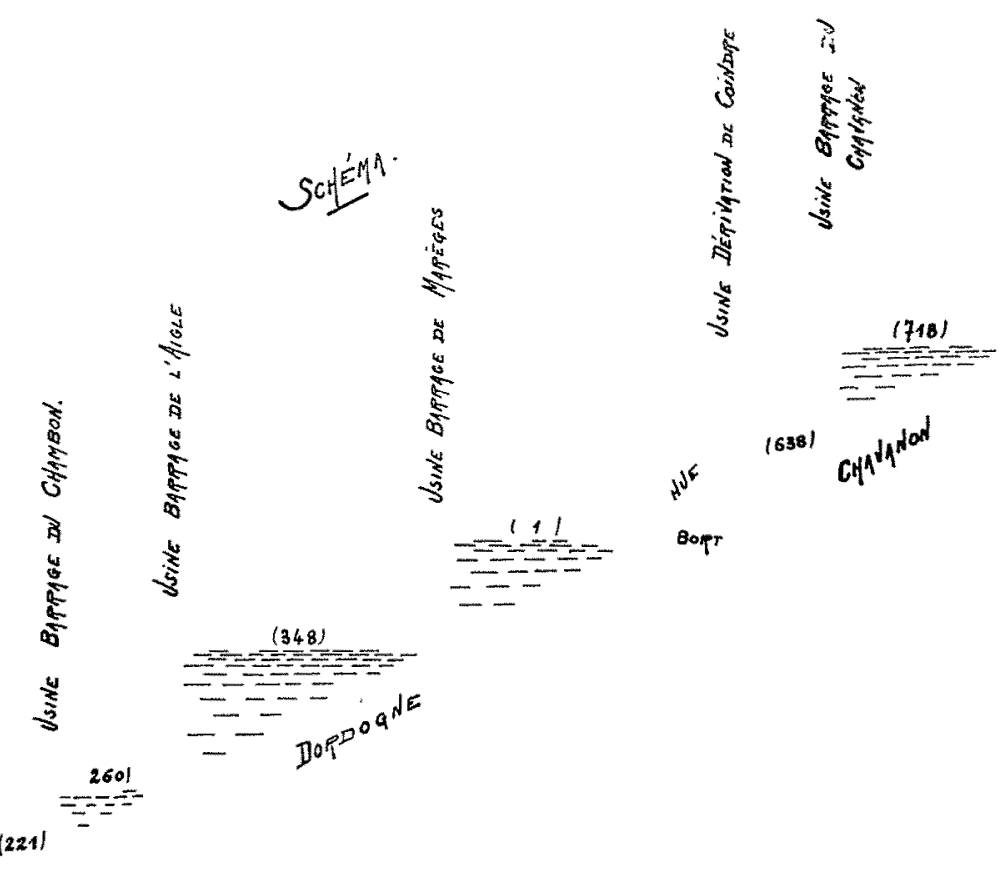

l'autre dite Moyenne Dordognè (M. D.) et comprenant les deux chutes de "l'Aigle " (usine-barrage de $88 \mathrm{~m}$. à $3 \mathrm{~km}$. 5 environ en aval du pont de Nauzenac) et du "Chambon " (usine-barrage de $39 \mathrm{~m}$. à $4 \mathrm{~km}$. environ en aval du pont du Chambon) (1).

L'étude (ainsi provisoirement simplifiée) est faite sur la base des débits mensuels moyens, donc "au mois le mois " et non " au jour le jour ". Elle est faite en "puissance moyenne continue ".

$1^{0}$ Dans une hypothèse $N^{0} 1$, le groupe $H$ D fonctionne suivant la consigne suivante : soit $\mathrm{P}$. la puissance totale moyenne continue du groupe et $\mathrm{H}$ la cote $\mathrm{N}$. G. F. en fin de mois dans le réser-

(1) Il va de soi que cêt exposé ne doit pas être envisagé comme étant autre chose qu'un choix d'excmples arbitraires destinćs seulement à fixer les idées; il ne préjuge en quoi que ce soit du schéma d'aménagement à adopter ou de l'ordre d'exécution des chutes, pas plus que ce que nous imaginons plus loin pour fixer les idées touchant une liaison avec le Rhône. 
voir du Chavanon. On tendra à réaliser successivement les condiLions classées ci-après par priorité : $\mathrm{P}$. $=18.500 \mathrm{kw} ; \mathrm{H}=\mathrm{f}(\mathrm{m})$ fonction définie ci-dessous (1) $; \mathrm{P} .=41.000 \mathrm{kw} \times 80 \% ; \mathrm{H}=$ $718 ; \mathrm{P} .=41.000 \mathrm{kw}$. Toujours et partout, dans toutes les hypothèses, entre différentes façons de satisfaire également à une condition, on choisira celle qui videra le moins les réservoirs ; on ne videra jamais Marèges que si le Chavanon a lui-même épuisé sa tranche utile et on remplira toujours Marèges par priorité. On ne videra d'ailleurs jamais Marèges pendant les mois autres qu'octobre inclus à février inclus et exclusivement pour réaliser la première des conditions. Enfin, de février inclus à septembre inclus, on se contentera exceptionnellement de $\mathrm{P}<18.500$ mais $>15.000$, dans la mesure où ce serait nécessaire pour que $\mathrm{H}$ ne soit pas $<\mathrm{l}(\mathrm{m})-12 \mathrm{~m}$. ; on descendrait au-dessous si c'était nécessaire pour que $P$. ne soit pas inférieur à 15.000 .

Nous ne dissimulons aucunement que cette consigne est le résultat de tâtonnements et d'ébauches faites sur les années écoulées (2), en sera-t-il jamais autrement d'une consigne quelconque et le passé n'est-il pas, en cette matière, le seul regard ouvert sur l'avenir ?... Telle qu'elle est, et en répétant bien que ('e n'est qu'une première approximation, elle répond cependant à la logicue. On a supposé que le principal des paramètres etait la puissance minimum à obtenir du groupe et en vue de laquelle on n'hésitcra pas à tirer sur les réservoirs ; une fois qu'on a tiré suffisamment pour obtenir cette puissance, faut-il avoir pour but le remplissage complet et doit-on ne rechercher une puissance plus forte qu'après avoir rempli tous les bassins ?... Il est, a priori tout au moins, assez normal d'envisager qu'à certaines époques de l'année plus rapprochées des pluies probables, on se contente d'atteindre une certaine cote dans tel réservoir, une certaine cote dans te! autre, aprc̀s avoir avant tout assuré la puissance minimum : ces cotes, d'ailleurs, étant, cela se conçoit fort bien a priori, variables suivant l'époque de l'année (et les sources d'énergie avec lesquelles on se couple). On conçoit également que la puissance minimum à réaliser soit atténuée par tolérance lorsque la situation des réservoirs se trouve exceptionnellement mauvaise ; on conçoit aussi que, lorsqu'on a assuré le remplissage partiel des réservoirs, on ne cherche à atteindre qu'un " palier " de la puissance la plus forte que puissent donner les armements, après quoi on remplira à fond les réservoirs avant de tendre vers la totalité de cette puissance maximum.

Les résultats de cette consigne sont donnés en traits pleins au graphique $\mathrm{A}$ ci-après .

$2^{0}$ Dans une hypothèse $N^{0} 2$, le groupe MD reçoit les débits du groupe HI fonctionnant dans l'hypothèse $\mathrm{N}^{0} 1$ et fonctionne suivant la consigne suivante: Soit $\mathrm{P}$ la puissance moyenne continue du groupe et $\mathrm{H}$ la cote N. G. F en fin de mois dans le réservoir de l'Aigle. On tendra à réaliser successivement les conditions classées ci-après par priorité : $\mathrm{P}=31.500 \mathrm{kw}$; $\mathrm{H}=\mathrm{f}(\mathrm{m})(3)$; $\mathrm{P}=68.500 \mathrm{kw} . \times 80 \% ; \mathrm{H}=348 ; \mathrm{P}=68.500 \mathrm{kw}$. De janvier inclus à scptembre inclus, on se contentera exceptionnellement de $\mathrm{P}<31.500$ mais $>26.000$, dans la mesure où ce serait nécessaire pour que $H$ ne soit pas $<\mathrm{f}(\mathrm{m})-15 \mathrm{~m}$.; toutefois, on descendrait au-dessous si c'était nécessaire pour que $\mathrm{P}$ ne soit pas inférieur à 26.000 .

(1) De janvier à décembre : $710-713-716-718-718-$ $718-715-711,50-708-708-708-708$.

(2) Il est cependant intéressant de noter qu'elle a ćté arrêtée en juillet 1924 et qu'elle n'est appliquée qu'au fur et à mesure à l'hiver 192.t-1925: les résultats en sont, jusqu'à ce jour, satisfaisants pour ledit hiver.

(3) De décembre à janvier : $340-343-346-348-348-$ $348-345-341,50-337-329-320-320$.
Les résultats de cette consigne sont donnés en traits pleins au graphique $B$ ci-après.

$3^{0}$ Dans une hypothèse $N^{0} 3$, le groupe HD est jumelé avec une puissance produite par le Rhône. On admettra (car il faut bien ici encore faire une hypothèse arbitraire à ce sujet pour fixer les idées, que la puissance moyenne continue sur le Rhône avec laquelle on échange de l'énergie, est donnée par la formule $1 / 3 \mathrm{R} \times 67 \times 7, \mathrm{R}$ étant, le débit mensuel moyen en mètres cubes à Génissiat quand ce débit est $<300 \mathrm{~m}^{3}$ et étant pris égal à $300 \mathrm{~m}^{3}$ le reste du temps (1). Ceci dit, la consigne est la suivante : Soit $\mathrm{P}$ la prissance moyenne totale continue de l'ensemble Dordogne et Rhône : on tendra à réaliser d'abord $\mathrm{P}=51.500$, puis $\mathrm{H}=718$; puis $\mathrm{P}=88.000$. On admettra toujours pour fixer les idées - que le contrat d'échange d'énergie attribue à la Dordogne les kw pris au Phône qui sont nécessaires à la régularisation de la Dordogne à $28.000 \mathrm{kw}$. et attribue au Rhône ceux pris à la Dordogne qui sont nécessaires à la régularisation du Rhône à $23.500 \mathrm{kw}$; on admettra ensuite que la Dordogne cède au Rhône les kw. au-dessus de $28.000 \mathrm{kw}$. qui peuvent compléter le Rhône à $37.500 \mathrm{kw}$.

Les résultats de l'application de cette consigne et de cette entente, pour la Dordogne, sont donnés en traits ponctués au graphique A. On voit combien cette conjugaison améliore la puissance minima et qu'elle modifie profondément le régime effectif de vidange des réservoirs de la Dordogne, le rôle utile de ceux-ci reste d'ailleurs considérable.

$4^{0}$ Dans une hypothèse $N^{0} 4$, le groupe MD reçoit les débits du groupe HD fonctionnant dans l'hypothèse $\mathrm{N}^{0} 3$ ci-dessus et fonctionne suivant une consigne analogue, avec un traité d'échange d'énergie analogue. Les résultats de l'application de cette consigne et de cette entente, pour la Dordogne, sont donnés en traits ponctués au graphique $B$.

$5^{0}$ Nous avons étudié sommairement, toujours par les mêmes méthodes, une hypothèse qui consisterait à faire fonctionner, sous une exploitation unique, d'une part l'ensemble HD + MD non conjugué avec le Rhône; d'autre part le même ensemble conjugué avec le Rhône. Il semble qu'on puisse ainsi augmenter, mais dans de très faibles proportions (en l'espèce moins de $5 \%$ ), la puissance minima régularisée. Quoi qu'il en soit, on voit que l'application de la méthode préconisée est une base tout à fait précise pour les ententes à interverir en cas d'exploitation unique d'un ensemble.d'usines appartenant à des concessionnaires différents, puisqu'elle permet à chaque moment de savoir ce qui revient à chacun dans le total de l'énergie produite : il suffit pour cela de calculer à chaque moment ce qu'aurait donné l'exploitation séparée et, s'il y a une bonification par le fait de l'exploitation unique, d'en faire bénéficier chacun proportionnellement. Ce dernier point nous a paru intéressant à signaler, car nous avons souvenir d'avoir vu discuter à perte de vue et sans aucun résultat d'ailleurs, sur les modalités de l'entente qui pourrait intervenir en pareil cas.

\section{Bordeaux, 20 avril 1925.}

P.-S. - Dans l'élaboration technique du présent travail, nous avons été puissamment aidé par l'étroite et précieuse collaboration de MM. Sarrat et Thibault, ingénieurs adjoints des T.P.E., Bouhant,'ancien élève de l'Ecole Polytechnique, et Martinet, ingénieur adjoint des T. P. E.

(Il doit être entendu que la présente note n'exprime que les idées personnelles de son auteur et n'engage en quoi que ce soit la pensée de l'Administration).

(1) Minimum de $R$ pendant la période envisagée: $140 \mathrm{~m}^{3}$. 